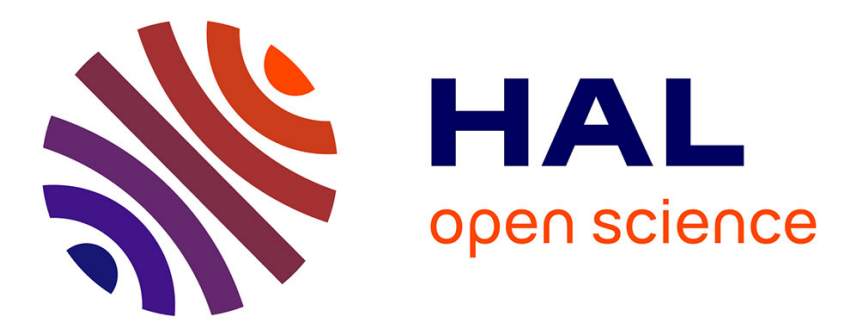

\title{
Découverte à La Réunion de Elaphoglossum multisquamosum (Dryopteridaceae), connue jusqu'alors comme endémique de Madagascar
}

Yannis Robert, Hermann Thomas, Jean Maurice Tamon, Germinal Rouhan

\section{- To cite this version:}

Yannis Robert, Hermann Thomas, Jean Maurice Tamon, Germinal Rouhan. Découverte à La Réunion de Elaphoglossum multisquamosum (Dryopteridaceae), connue jusqu'alors comme endémique de Madagascar. Candollea, 2021, 76 (1), pp.99-104. 10.15553/c2021v761a10 . mnhn-03174090v2

\section{HAL Id: mnhn-03174090 \\ https://hal-mnhn.archives-ouvertes.fr/mnhn-03174090v2}

Submitted on 24 Mar 2021

HAL is a multi-disciplinary open access archive for the deposit and dissemination of scientific research documents, whether they are published or not. The documents may come from teaching and research institutions in France or abroad, or from public or private research centers.
L'archive ouverte pluridisciplinaire HAL, est destinée au dépôt et à la diffusion de documents scientifiques de niveau recherche, publiés ou non, émanant des établissements d'enseignement et de recherche français ou étrangers, des laboratoires publics ou privés. 


\title{
Découverte à La Réunion de Elaphoglossum multisquamosum (Dryopteridaceae), connue jusqu'alors comme endémique de Madagascar
}

\author{
Yannis Robert, Hermann Thomas, Jean Maurice Tamon \& Germinal Rouhan
}

\begin{abstract}
ROBERT, Y., H.THOMAS, J.M. TAMON \& G. ROUHAN (2021). Discovery in La Reunion Island of Elaphoglossum multisquamosum (Dryopteridaceae), known so far as endemic to Madagascar. Candollea 76: 99-104. In French, English and French abstracts. DOI: http://dx.doi.org/10.15553/ c2021v761a10

A species of the fern genus Elaphoglossum Schott ex J. Sm. (Dryopteridaceae) has been discovered in La Réunion. It is ascribed to Elaphoglossum multisquamosum Bonap., described and known so far as endemic to Madagascar. Taking advantage of this new record, Elaphoglossum multisquamosum is reinstated as a species distinct from the Malagasy endemic Elaphoglossum poolii (Baker) Christ. Morphologically close to Elaphoglossum poolii that is often observed in sympatry in Madagascar, Elaphoglossum multisquamosum has often been treated as a variety of Elaphoglossum poolii or even placed in synonymy of the latter species. We provide a novel, fully detailed description of Elaphoglossum multisquamosum, all critical characters supporting it as distinct from Elaphoglossum poolii, and a comparative map of distribution for the two species in Madagascar and La Réunion. Finally, the name Elaphoglossum multisquamosum is lectotypified.
\end{abstract}

\section{Résumé}

ROBERT, Y., H.THOMAS, J.M.TAMON \& G. ROUHAN (2021). Découverte à La Réunion de Elaphoglossum multisquamosum (Dryopteridaceae), connue jusqu'alors comme endémique de Madagascar. Candollea 76: 99-104. En français, résumés anglais et français. DOI: http://dx.doi.org/10.15553/ c2021v761a10

Une espèce de fougère du genre Elaphoglossum Schott ex J. Sm. (Dryopteridaceae) a été découverte à La Réunion et identifiée comme Elaphoglossum multisquamosum Bonap., décrite et connue jusqu'alors comme endémique de Madagascar. A l'occasion de cette découverte, Elaphoglossum multisquamosum est confirmée comme une espèce distincte de l'endémique malgache Elaphoglossum poolii (Baker) Christ. Morphologiquement proche de Elaphoglossum poolii qui se trouve souvent en sympatrie à Madagascar, Elaphoglossum multisquamosum a ainsi souvent été considérée au rang variétal de Elaphoglossum poolii ou même placée en synonymie de cette dernière. Nous fournissons une nouvelle description morphologique détaillée de Elaphoglossum multisquamosum, tous les caractères nécessaires la soutenant comme distincte de Elaphoglossum poolii, ainsi qu'une carte de répartition comparative pour les deux espèces à Madagascar et à La Réunion. Enfin, le nom Elaphoglossum multisquamosum est lectotypifié.

\section{Keywords}

PTERIDOPHYTES - DRYOPTERIDACEAE - Elaphoglossum - Madagascar - Mascarenes - Taxonomy - Typification

Adresses des auteurs:

YR, JMT: Association des naturalistes de Bourbon, 107 chemin Dioré, 97440 Saint André, La Réunion.

HT: Parc national de La Réunion, 258 rue de la république, 97431 La Plaine des Palmistes, La Réunion.

GR: Institut de Systématique, Évolution, Biodiversité (ISYEB), Muséum national d'Histoire naturelle, Sorbonne Université, École Pratique des Hautes Etudes, Université des Antilles, CNRS, 57 rue Cuvier CP39, 75005 Paris, France. E-mail: rouhan@mnhn.fr 


\section{Introduction}

Le genre Elaphoglossum Schott ex J. Sm. (Dryopteridaceae) est le deuxième genre de fougères le plus diversifié (après Asplenium L.) avec sans doute plus de 600 espèces (PPG I, 2016). Majoritairement épiphytes, les espèces se répartissent dans la plupart des forêts humides tropicales. L'ouest de l'Océan Indien pourrait être le second centre de diversité après les néotropiques qui abritent $75 \%$ des espèces (RouHAN et al., 2004). Le genre est représenté dans la région malgache par environ 80 espèces (Rouhan, 2020; G. Rouhan, données non publiées), dont en particulier 22 espèces ou hybrides présents dans l'archipel des Mascareignes et 15 à La Réunion (LoREncE \& Rouhan, 2004, 2008).

Le genre est bien distinct mais sa taxonomie est complexe car la plupart des espèces sont à première vue morphologiquement uniformes: presque toutes sont en effet distinctes par une méristèle ventrale transversale et allongée dans les rhizomes, des frondes simples, des nervures libres, des frondes stériles et fertiles dimorphes, et des sores acroctichoïdes (RouHan et al., 2004; Moran et al., 2010). Contrairement à la plupart des autres fougères, les frondes fertiles de Elaphoglossum ne sont pas cruciales pour la taxonomie du genre, qui se fonde essentiellement sur les écailles des rhizomes et frondes. Le genre est subdivisé en 7 sections morphologiques (Mickel \& Atehortúa, 1980; Lóriga et al., 2014) largement soutenues par les phylogénies moléculaires (RouHAN et al., 2004; LóRIGA et al., 2014; VAsco et al., 2015) et les spores (Moran et al., 2007). Cinq des sept sections du genre sont représentées dans l'Océan Indien.

Lors d'un inventaire botanique à La Réunion en 2010, nous avons observé et récolté une nouvelle espèce, appartenant à Elaphoglossum sect. Lepidoglossa Christ: E. multisquamosum Bonap. Cette espèce était jusqu'alors considérée comme endémique de Madagascar et souvent traitée en synonymie de l'endémique malgache $E$. poolii (Baker) Christ (CHristensen, 1932; Roux, 2009; APD, 2021) avec laquelle elle est parfois sympatrique à Madagascar.

Cette note a trois objectifs: a) fixer l'application du nom Elaphoglossum multisquamosum, en le lectotypifiant; b) préciser la description morphologique de E. multisquamosum sur l'ensemble de son aire de répartition actualisée; c) clarifier la distinction morphologique entre E. multisquamosum et E. poolii qui sont fréquemment confondues sur le terrain et dans les collections d'herbiers.

\section{Discussion}

L'espèce de Elaphoglossum observée et récoltée à La Réunion aux alentours du col du Taïbit appartient à la section Lepidoglossa. Cette section est globalement caractérisée par des taxons à limbe écailleux, ou sans écailles mais généralement avec des ponctuations glutineuses, des écailles qui sont planes

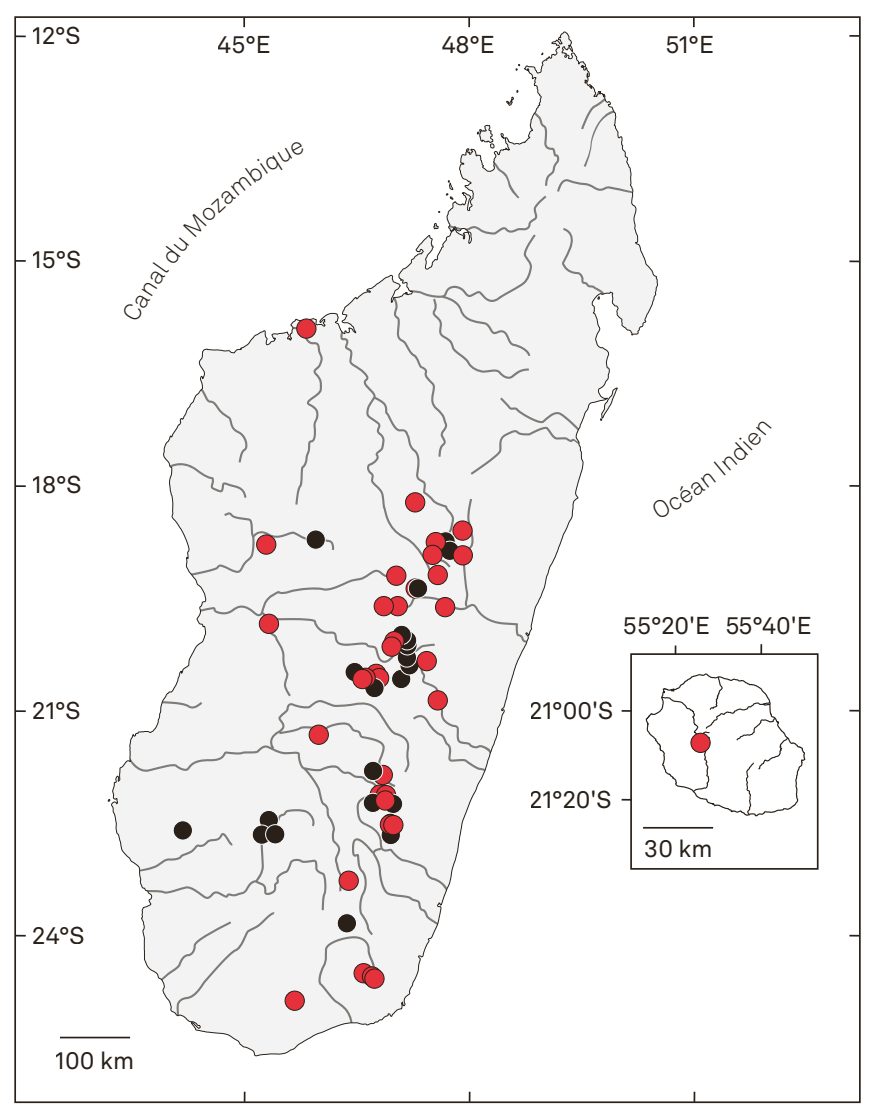

Fig. 1. - Carte de répartition de Elaphoglossum multisquamosum Bonap. (cercles rouges) et E. poolii (Baker) Christ (cercles noirs) à Madagascar et La Réunion.

ou à base hémi-infundibuliforme sur les frondes, et des rhizomes écailleux ou glabres, glutineux ou non.

Les sept taxons de la section Lepidoglossa actuellement reconnus à La Réunion (LoRence \& Rouhan, 2008) sont Elaphoglossum heterolepis (Fée) T. Moore, E. xheterophlebium Lorence, E. lancifolium (Desv.) C.V. Morton, E. richardii (Bory ex Fée) Christ, E. rufidulum (Willd. ex Kuhn) C. Chr., E. splendens (Bory ex Willd.) Brack. et E. stipitatum (Bory ex Fée) T. Moore. L'espèce de Elaphoglossum du col du Taïbit se distingue de ces sept espèces par l'association de caractères morphologiques suivante: les écailles du rhizome sont en général brun clair mat, les frondes densément couvertes d'écailles ciliées unicolores et dressées en toutes directions sur le pétiole, et les frondes fertiles sont plus longues que les stériles. Par conséquent, cette espèce est nouvelle pour La Réunion. Une étude exhaustive de l'ensemble de la diversité des espèces décrites en Afrique, dans l'Océan Indien et dans les îles australes du sud de l'Océan Indien a permis d'identifier l'espèce nouvelle de La Réunion comme étant Elaphoglossum multisquamosum, décrite et connue jusqu'alors comme une endémique de Madagascar. Dans la clé de détermination des Elaphoglossum des Mascareignes (LoRence \& Rouhan, 


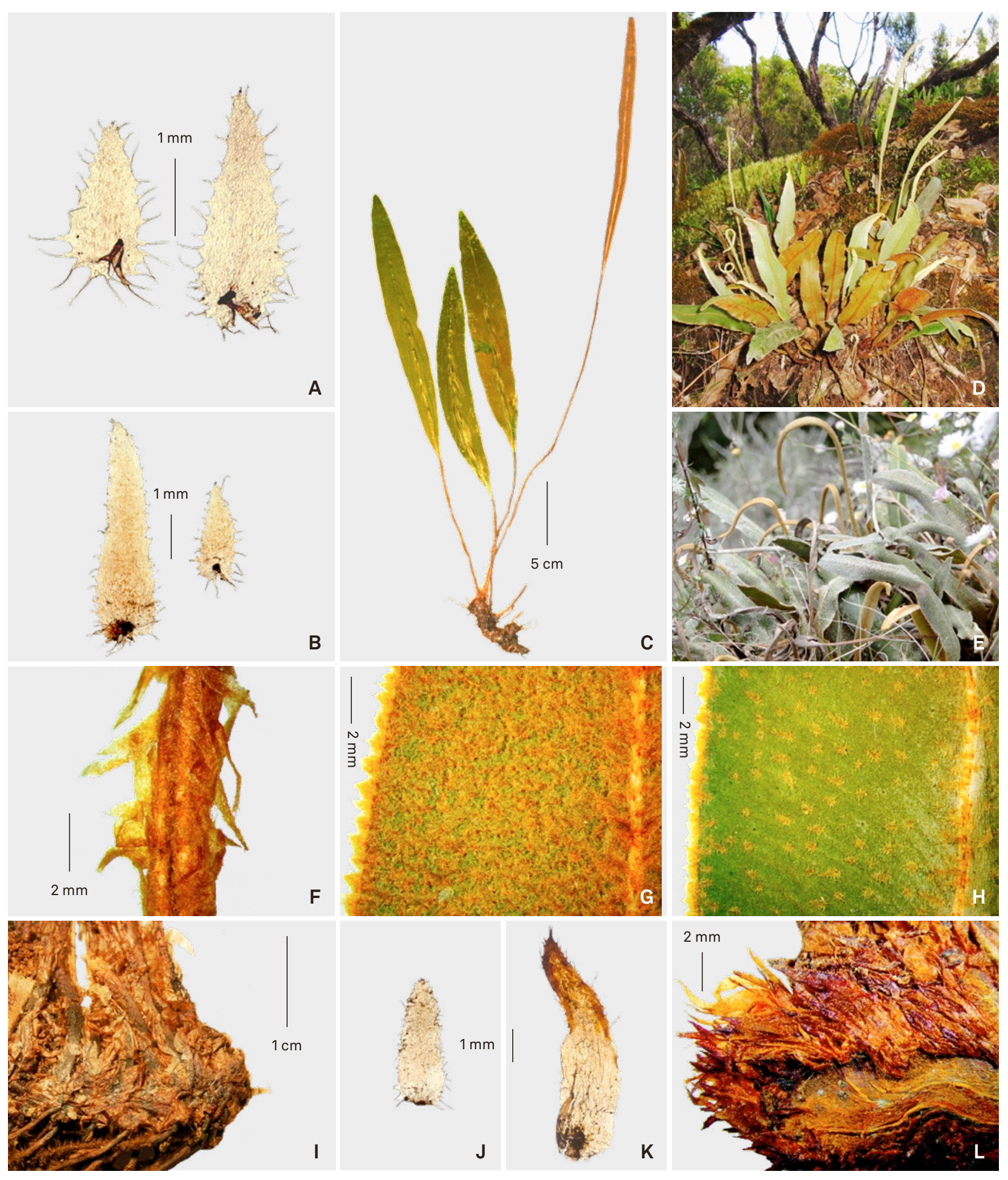

Fig. 2. - Elaphoglossum multisquamosum Bonap. A. Ecailles du limbe; B. Ecailles du pétiole; C. Plante entière fertile; D-E. Aspect général; F. Pétiole; G. Détail du limbe stérile, face inférieure; H. Détail du limbe stérile, face supérieure; I. Rhizome; J-K. Ecailles du rhizome; L. Rhizome. [A-B, I-J: Rouhan 443, P00749412; C: Waterlot s.n., P01359928; D: Rouhan 473, P00749446; E: Robert 1335, P00749889; F-H: Proisy 249, P00636592; K-L: Andriamihajarivo 45, P01031713] [Photos: A-D, F-L: G. Rouhan; E: Y. Robert] 
2008), E. multisquamosum sortirait au couplet 23 , qui devrait être modifié comme suit:

23a. Écailles du rhizome le plus souvent brun clair, mates, scarieuses E. multisquamosum

23b. Écailles du rhizome brun foncé à marron foncé, brillantes, raides et cassantes [23bis]

[23bis a]. Feuilles stériles complètement recouvertes sur les deux faces du limbe d'une couche d'écailles brun rougeâtre avec souvent des écailles brun foncé à noir le long de la nervure médiane abaxiale

20. E. splendens

[23bis b]. Feuilles stériles portant des écailles brun rougeâtre pâle, principalement le long des nervures du limbe 16. E. rufidulum

Elaphoglossum multisquamosum a été traité au rang d'espèce à part entière (BONAPARTE, 1915), ou de variété de l'espèce endémique malgache $E$. poolii dans la Flore de Madagascar et des Comores (Tardieu-Blot, 1960), ou même en synonymie de E. poolii (e.g. Christensen, 1932; APD, 2021). Pour ajouter à la confusion, Roux (2009: 130) accepte ce taxon au rang spécifique puis deux pages plus loin comme variété de E. poolii (Roux, 2009: 131). Elaphoglossum poolii et E. multisquamosum sont en effet deux taxons morphologiquement proches par leurs écailles du rhizome brun clair mat, et leurs écailles du limbe ciliées, ovées à lancéolées et mesurant $1,5-4 \times 0,5-1,5 \mathrm{~mm}$. De plus, les deux espèces sont le plus souvent terrestres ou épilithes, à la différence de la grande majorité des espèces du genre qui sont épiphytes et exceptionnellement terrestres. Enfin, comme elles se trouvent assez souvent en sympatrie (Fig. 1), elles sont fréquemment confondues sur le terrain et se trouvent parfois en mélange dans les récoltes (e.g. Humbert 3776, Humbert 28227, Labat et al. 3662) et donc montées ensemble en mélange sur des planches d'herbiers (P [P00611249 vs. P00915739; P00915740 vs. P00151004; P00915738 vs. P01556626]). Toutefois, E. multisquamosum se distingue toujours par les frondes stériles moins longuement pétiolées $(<1 / 2$ de la longueur de la fronde vs. $>1 / 2$ pour $E$. poolii), les limbes étroitement elliptiques ou oblancéolés (vs. oblongs pour E. poolii), les écailles de la base du pétiole qui ne sont ni antrorses ni engainantes, et par les écailles du limbe plus étroites $(0,5-1 \mathrm{~mm}$ vs. $1-1,5 \mathrm{~mm}$ pour E. poolii) à cils marginaux moins nombreux $(10-15(-20)$ paires vs. (10-)20-40 pour E. poolii) et plus longs (aussi longs que la largeur du corps de l'écaille vs. plus courts que la largeur du corps de l'écaille pour $E$. poolii).

En comparaison avec les espèces se trouvant en dehors de son aire de répartition, E. multisquamosum est morphologiquement plus proche de E. succisifolium (Thouars) T. Moore des îles australes du sud de l'Océan Indien et de Tristan da Cunha (Atlantique Sud), mais les deux espèces diffèrent clairement par l'ensemble de leurs écailles, et cette distinction est bien soutenue par la génétique (Rounan et al., 2004).

\section{Taxonomie}

Elaphoglossum multisquamosum Bonap. in Notes Ptéridol. 1: 67.1915 (Fig. 2).

$\equiv$ Elaphoglossum poolii var. multisquamosum (Bonap.) Tardieu in Humbert, Fl. Madagascar 5(2): 54. 1960.

Lectotypus (hic designatus): Madagascar. Reg. Analamanga [Prov. Antananarivo]: «Halfy», Ilafy, [18 51 "S 47³3,5"E], I.1906, D'Alleizette 42 (P [P00466228]!). Syntypus: Madagascar. Reg. Analamanga [Prov. Antananarivo]: Ambohimanga, [18 $45^{\prime} \mathrm{S} 47^{\circ} 33^{\prime} \mathrm{E}$ ], 12.VI.1905, D'Alleizette 167 (P [P00466229, P00466230]!).

Rhizomes courtement rampant parfois ascendant, 3,5-6,5 mm de diamètre, ramifiés, plus ou moins glutineux, densément couverts d'écailles au moins à l'apex (Fig. 2I, $\mathrm{L}$ ); écailles du rhizome peu à entièrement appliquées (et alors non distinctes les unes des autres), ovées à lancéolées, $2,5-6(-10) \times 1-2 \mathrm{~mm}$, la plupart brun clair mat, scarieuses, bases cordées, apex cunéiformes à atténués, marges subentières avec des glandes sessiles et des cils courts $(<0,2 \mathrm{~mm})$, parfois avec d'autres écailles dont les marges ciliées sont marron brillant (Fig. 2J-K). Frondes stériles dressées à étalées (Fig. 2D, E), insérées sur au moins deux rangs peu distincts, distantes de moins de $5 \mathrm{~mm}$, longues de $8-47 \mathrm{~cm}$. Pétioles longs de 2-16 cm, représentant généralement moins de la moitié de la longueur de la fronde, $0,5-1,5 \mathrm{~mm}$ de diamètre, densément couverts d'écailles; écailles du pétiole dressées en toutes directions, lancéolées, 2-6 × 1-1,5 mm, roux clair mat, translucides, scarieuses, bases pseudo-peltées, apex cunéiformes à acuminés, marges sub-entières à ciliées avec plus de 10 cils de chaque côté, courts $(<0,5 \mathrm{~mm})$ et plus ou moins droits (Fig. 2B, F). Limbe stérile étroitement elliptique ou oblancéolé, rarement linéaire, 6-32 × 1,3-4 cm, base cunéiforme à atténuée, apex arrondi ou aigu, texture chartacée à coriace, les deux faces et les marges densément écailleuses jusqu'à masquer parfois la face inférieure du limbe, face supérieure glabrescente (Fig. 2G-H); écailles du limbe imbriquées, ovées à lancéolées, 1,5-3 $\times 0,5-1 \mathrm{~mm}$, rousses, souvent blanches sur la face supérieure du limbe, translucides, scarieuses, base cordée à hémiinfundibuliforme, apex obtus, arrondi ou cunéiforme, marges ciliées avec 10-15 cils aciculaires de chaque côté, souvent aussi longs que la largeur du corps de l'écaille (Fig. 2A); nervure médiane proéminente et arrondie sur la face inférieure, aplatie sur la face supérieure; nervures latérales parfois légèrement proéminentes sur la face inférieure, simples ou 1-2-bifurquées, libres jusqu'à leur apex. Frondes fertiles plus longues que les stériles, atteignant $60 \mathrm{~cm}$, plus étroites $<1,5 \mathrm{~cm}$, avec le pétiole $1 / 2-3 / 5$ de la fronde (Fig. 2C).

Distribution et écologie. - Elaphoglossum multisquamosum est présent à Madagascar au centre et au sud (et une récolte à 
Mahajanga), dans les domaines phytogéographiques du Centre et de l'Ouest (Humbert, 1955), à 100-2650 m d'altitude. Elle pousse dans la forêt sempervirente résiduelle, le long des cours d'eau et dans les ravins où elle est peu fréquente. C'est une plante épilithe ou terrestre, rarement épiphyte.

A la Réunion, une seule station est connue, dans le cirque de Cilaos, à plus de 1950 m, au niveau de la Plaine aux Fraises sous le rempart des trois Salazes. La plante s'y développe sur une falaise rocheuse avec d'autres herbacées communes des prairies d'altitude, dans une zone de transition entre «forêt de bois de couleurs d'altitude» (Dombeya spp.) et végétation éricoïde (Erica spp.). Selon la typologie des habitats "Corine Biotope» (Strasberg et al., 2010), le code habitat [49.214] de la station est qualifié de forêt semi-sèche de transition vers l'étage mésotherme des cirques et grandes vallées sous le vent, étage à Dombeya spp. A La Réunion, l'espèce pourrait être menacée par la présence de l'exotique envahissante Erigeron karvinskianus DC. (C. Lavergne, comm. pers.).

Notes. - Les trois spécimens types (deux récoltes) représentent tous la même espèce, Elaphoglossum multisquamosum, et deux portent la mention «Original» de la main de Bonaparte, auteur de l'espèce. Nous avons choisi comme lectotype le spécimen [P00466228] avec deux plantes fertiles.

Elaphoglossum multisquamosum est polymorphe à Madagascar, avec deux morphologies extrêmes, la première à rhizome très glutineux couvert d'écailles appliquées et la seconde à rhizome peu glutineux couvert d'écailles plutôt dressées dont certaines présentent une marge marron brillant; cependant, les intermédiaires entre ces morphologies existent. La forme de l'apex du limbe et la taille des écailles du limbe sont aussi variables.

Spécimens observés. - Madagascar. Reg. Alaotra-Mangoro [Prov. Toamasina]: Analanomby, forêt du N du pays Sihanaka, 2.IX.1937, Jard. Bot. 3016 (P). Reg. Amoron'i Mania Prov. Fianarantsoa]: au N d'Ambatoandrano, 7.II.2001, Andriamihajarivo 45 (P); env. d'Ambatofinandrahana, 21.II.1938, Decary 13200 (P); montagnes à l'W d'Itremo (W Betsileo), 17.I-22.IV.1955, Humbert 28227 p.p. (P [P00915740, P01556626]); juste à l'E du col d'Itremo, 26.XI.1993, Labat 2439 (P); Fiadanana, Ilaka, Ambositra, 20.III.1960, Peltier 2145 (P); Fianarantsoa, Mania, IV.1919, Perrier de la Bâtbie 12575 (P). Analamanga [Prov. Antananarivo]: Mandraka, III.1961, Bosser 15215 p.p. (P [P00611253]); Ambohidempona, 26.VII.1911, Colin s.n. (P); Vodivato, montagne, 5.XI.1946, Cours 3060 (P); Mandraka, VIII.1906, D'Alleizette 213 (P); Antananarivo, IX.1921, Decary s.n. (P00611275); Ambohimanga, 27.III.1921, Decary 489 (P); Tananarive (fossés du Fort Voyron), 11.III.1928, Decary 6168 (P); Andramasina, 30 km SSE Tananarive, 17.II.1960, Leandri 3088 (P); Ambohimanga, à $20 \mathrm{~km}$ de Tananarive, 1919, Rabarijaona 9 (P); RS d'Ambohitantely, 8.XII.1997, Rakotondrainibe 4445 (P); forêt de la Mandraka, 17.VIII.1906, Rotereau s.n. (P [2 planches]); Tananarive, 1.IV-30.IV.1915, Waterlot s.n. (P [6 planches]). Reg. Androy [Prov. Toliara]: Ambohibe, s.d., Decary 4217 (P). Reg. Anosy [Prov. Toliara]: Mt. Itrafanaomby (Ankazondrano) et ses contreforts SW (Haut Mandrare), XII.1933, Humbert 13521 (P); massif de l'Andohahela (SE), I.1934, Humbert 13589 (P); R.N.I. d'Andohahela, versant E et sommet du Trafon'omby, 23.XI.1995, Rakotondrainibe 3186 (P [P00067309]); ibid. loco, versant du Trafon'omby, 28.XI.1995,
Rakotondrainibe 3216 (P). Reg. Boeny [Prov. Mahajanga]: Mt Namiaka (Boeny E), VIII.1905, Perrier de la Bâthie 7454 (P). Reg. Bongolova [Prov. Antananarivo]: forêt de Bongolava (100 km W Tsironomandidy vers Morafenobe), 17.VII.1974, Cremers 3251 (P); Mt Tsitondriana, 15.IV.1941, Jard. Bot. 4757 (P). Reg. Ihorombe [Prov. Fianarantsoa]: Andringitra, distr. Ambalavao autour du lieu-dit Anosibe, 9.V.1957, Cours 5172 (P); Andringitra, distr. Ambalavao au pied du Tsoraha, 10.V.1957, Cours 5179 (P); Pic d'Ivohibe, 23.IX.1926, Decary 5288 (P); Andringitra AP, II.1995, Goodman s.n. (P); Pic Boby, s.d., Homolle 1185 (P); Pic d'Ivohibe (Bara), 5.XI.1924, Humbert 3276 (P); ibid. loco, vallée de la Riambava et de l'Antsifotra, 27.XII.1924, Humbert 3776 (P); massif du Kalambatitra (centre-sud), Mt. Analatsitendrika, XII.1933, Humbert 11931 (P); Pic d'Ivohibe, haute vallée de l'Andranomainty, 23.IV.2005, Janssen 2810 p.p. (P [P00590873]); Ambalavao, Anara, Réserve d'Anja, 22.III.2006, Labat 3662 p.p. (P [P00533707]); massif d'Andringitra, IX.1911, Perrier de la Bâthie 7563 (P); ibid. loco, III.1921, Perrier de la Bâthie 13697 (P); RS d'Ivohibe, à $6,5 \mathrm{~km}$ à l'ESE du village d'Ivohibe, près de la source de la riv. Andranomainty, 25.X.1997, Rakotondrainibe 4241 (P); ibid. loco, 25.X.1997, Rakotondrainibe 4243 (P); PN de l'Andringitra, forêt de Riambavy, 17.XI.2004, Rouban 439 (P), Rouban 443 (P); ibid. loco, forêt de Ramiova, 17.XI.2004, Rouban 449 (P), Rouban 450 (P); ibid. loco, forêt de Anjavidilava, 19.XI.2004, Rouban 471 (P), Rouban 472 (P), Rouban 473 (P), Rouban 474 (P); RS du Pic d'Ivohibe, versant S au S du sommet, forêt dite du «plateau du Pic», 16.XI.2011, Rouhan 1397 (P). Reg. Itasy [Prov. Antananarivo]: Ankaratra, Antsahapandrano, 10.II.1942, Decary 17638 (P); Massif de l'Ankaratra, XII.1913, Perrier de la Bâthie 7613 (P); Ankaratra, IV.1980, Proisy 225 (P); forêt de Manjakatompo, versant E de l'Ankaratra, VII.1953, Service Forestier 58 (P). Reg. Vakinankaratra [Prov. Antananarivo]: summit Ibity, 5.II.2003, Birkinshaw 1193 (P); Manjakatompo, versant SE, 10.XI.1983, Rakotondrainibe 766 (P); sur la crête du Mt Ibity, 21.XI.1912, Viguier 1453 (P); massif du Vakinankaratra, 25.XI.1912, Viguier 1615bis (P); ibid. loco, coteaux boisés près de Tsinjoarivo, 30.XI.1912, Viguier 1884 (P). Reg. Vatovavy-Fitovinany [Prov. Fianarantsoa]: Ambohimanga Sud, V.1980, Proisy 249 (P). Sine loco: 19.IX.1917, D'Alleizette $212 \mathrm{~m}$ p.p. (P [P00611279]); s.d., Homolle 1243 (P).

La Réunion: Cirque de Cilaos, plaine aux Fraises sous l'arrête des Salazes, 29.V.2010, Robert 1335 (P, herbier privé Y. Robert).

\section{Remerciements}

Nous remercions l'Association des naturalistes de Bourbon dont les prospections de taxons rares permettent aussi des découvertes pour l'île comme Elaphoglossum multisquamosum. Nous remercions le Conservatoire Botanique National de Mascarin pour sa collaboration au long cours, et son Président de Conseil Scientifique, Vincent Boullet, pour ses travaux sur l'Index des Trachéophytes de La Réunion. Les permis de recherche à Madagascar ont été accordés par le Ministère de l'Environnement, et du Développement Durable et Madagascar National Parks (70/19/MEDD/SG/DGF/DSAP/SCB.Re, 207/15/MEEMF/SG/DGF/DAPT/SCBT, 199/15/MEEMF/ SG/DGF/DAPT/SCBT, 241/11/MEF/SG/DGF/DCB.SAP/ $\mathrm{SCB})$, et les recherches de terrain ont bénéficié du concours du CNRE, MBG et PBZT. Ce manuscrit a bénéficié des relectures constructives de Martin Callmander, Laurent Gautier et Pete Phillipson. 


\section{Références}

APD (2021). African Plant Database. Conservatoire et Jardin botaniques de la Ville de Genève et South African National Biodiversity Institute, Pretoria. [http://africanplantdatabase.ch]

Bonaparte, R. (1915). I. Plusieurs régions; plusieurs collecteurs. Herbier du Prince Bonaparte. Notes Ptéridol. 1: 47-72.

Christensen, C. (1932). The Pteridophyta of Madagascar. Dansk Bot. Ark. 7: [I]-XV, [1]-253.

Humbert, H. (1955). Les territoires phytogéographiques de Madagascar. Année Biol. 31: 439-448.

Lorence, D.H. \& G. Rouhan (2004). A revision of the Mascarene species of Elaphoglossum (Elaphoglossaceae). Ann. Missouri Bot. Gard. 91: 536-565.

Lorence, D.H. \& G. Rouhan (2008). Lomariopsidaceae. In: BAdré, F. (ed.), Fl. Mascareignes. Ptéridophytes, 1. Psilotacées à 26. Marsileacées.

Lóriga, J., A. Vasco, L. Regalado, J. Heinrichs \& R.C. Moran (2014). Phylogeny and classification of the Cuban species of Elaphoglossum (Dryopteridaceae), with description of Elaphoglossum sect. Wrightiana sect. nov. Pl. Syst. Evol. 300: 937-951.

Mickel, J.T. \& L. Atehortúa (1980). Subdivision of the genus Elaphoglossum. Amer. Fern J. 70: 47-68.

Moran, R.C., J.G. Hanks \& G. Rouhan (2007). Spore morphology in relation to phylogeny in the fern genus Elaphoglossum (Dryopteridaceae). Int. J. Pl. Sci. 168: 905-929.
Moran, R.C., J.G. Hanks, P. Labiak \& M. Sundue (2010). Perispore morphology of bolbitidoid ferns (Dryopteridaceae) in relation to phylogeny. Int. J. Pl. Sci. 171: 872-881.

PPG I [Pteridophyte Phylogeny Group] (2016). A communityderived classification for extant lycophytes and ferns. J. Syst. Evol. 54: 563-603.

Rouhan, G. (2020). Synoptic revision of the fern genus Elaphoglossum Schott ex J.Sm. (Dryopteridaceae) in Madagascar, with the description of 23 new taxa, all but one endemic. PeerJ 8: e10484. DOI: https://peerj.com/articles/10484

Rouhan, G., J.-Y. Dubuisson, F. Rakotondrainibe, T.J. Motley, J.T. Mickel, J.-N. Labat \& R.C. Moran (2004). Molecular phylogeny of the fern genus Elaphoglossum (Elaphoglossaceae) based on chloroplast non-coding DNA sequences: Contributions of species from the Indian Ocean area. Molec. Phylogen. Evol. 33: 745-763.

Roux, J.P. (2009). Synopsis of the Lycopodiophyta and Pteridophyta of Africa, Madagascar and neighbouring islands. Strelitzia 23.

Strasberg, D., J. Dupont \& J.-C. Rameau (2010). Typologie des milieux naturels et des habitats de La Réunion, 2000. DIREN Réunion / Université de la Réunion. [Révision de février 2010 par F. Picot \& M. Saliman].

Tardieu-blot, M.-L. (1960). Polypodiaceae (s.lat.) (BlechnaceaePolypodiaceae) (s.str.). In: Humbert, H. (ed.), Fl. Madagascar Comores 5(2).

Vasco, A., J. Lóriga, G. Rouhan, B.A. Ambrose \& R.C. Moran (2015). Divided leaves in the genus Elaphoglossum (Dryopteridaceae): a phylogeny of Elaphoglossum section Squamipedia. Syst. Bot. 40: 46-55. 